\title{
Depressionsbehandlung heute
}

\author{
Dietmar Winkler, Edda Pjrek, Siegfried Kasper \\ Klinische Abteilung für Allgemeine Psychiatrie, Medizinische Universität Wien
}

psychoneuro 2005; 31 (10): 495-498

$\mathrm{D}$ epressionen sind psychischer Schmerz, und schwere depressive Episoden gehören wahrscheinlich zu den unangenehmsten Erkrankungen, an denen Menschen leiden können (3). Betroffene beschreiben die Qualen, die sie im Rahmen von Depressionen erleiden als weit gravierender als jegliche nur erdenkliche körperliche Beschwerden. Der englische Autor Samuel Johnson (18. Jahrhundert), der in der Mitte seines Lebens unter schweren Depressionen litt, schrieb, dass er sich für seine psychische Gesundheit gerne Gliedmaßen amputieren ließe.

\section{Epidemiologie}

Depressive Erkrankungen haben weltweit eine überragende epidemiologische Bedeutung: die Lebenszeitprävalenz beträgt zwischen 15 und 20\%, wobei Frauen etwa doppelt so häufig betroffen sind (9). Laut der Weltgesundheitsorganisation (WHO) wird die Depression bis zum Jahr 2020 in der westlichen Welt der führende Grund für Invalidität und vorzeitigen Tod sein (13). Die sozioökonomischen Folgen von Depressionen sind enorm, das Leiden der Patienten tangiert auch deren Familien und Freunde; die Folgekosten durch die Erkrankung zum Beispiel durch Ausfall der Arbeitskraft haben einen gravierenden Einfluss auf die Wirtschaft. Depression ist eine potenziell lebensbedrohliche Erkrankung: $10-15 \%$ der stationär aufgenommenen depressiven Patienten sterben unbehandelt an Suizid (1). Vor diesem Hintergrund ist es verständlich, dass die adäquate Thera-

Depressionen sind die häufigste psychiatrische Erkrankung. Rund 15 bis 20\% aller Menschen leiden mindestens einmal in ihrem Leben an einer depressiven Episode. Zur Therapie schneidet eine Kombination aus psychotherapeutischer und medikamentöser Behandlung besser ab als die jeweilige Monotherapie. Neue pharmakologische Optionen bieten der selektive Serotoninund Noradrenalin-Wiederaufnahmhemmer Duloxetin sowie der enantiomerenreine selektive Serotonin-Wiederaufnahmehemmer Escitalopram. Bei bipolaren Depressionen sollte bei der Behandlung berücksichtigt werden, dass Antidepressiva einen Switch in die Manie auslösen können, weswegen gegebenenfalls eine phasenprophylaktische Therapie mit Stimmungsstabilisierern eingesetzt werden sollte.

pie depressiver Erkrankungen von überwältigender Bedeutung ist.

\section{Womit und wie lange behandeln?}

Prinzipiell können vom behandelnden Arzt sehr verschiedenartige Therapieformen angeboten werden: antidepressive Psychopharmakotherapie, Psychotherapie und die heterogene Gruppe nicht-pharmakologischer Verfahren, zu denen unter anderem Elektrokrampftherapie, transkranielle Magnetstimulation (2) Lichttherapie (besonders bei der saisonal abhängigen Depression vom Herbst-Wintertyp) sowie Schlafentzugstherapie zu zählen sind.

Für viele Patienten stellt sich bei der Eröffnung der Diagnose die Frage: Psychotherapie oder Medikamente? Bei leichter bis mittelschwerer Depression gibt es keinen Beleg, dass die Pharmakotherapie in der Akuttherapie wirksamer als die Psychotherapie wäre (4). Deshalb entscheidet hier primär der Wunsch des (informierten) Patienten; Psychotherapie setzt aber Kooperationsbereitschaft und -fähigkeit voraus. Psychotherapiemethoden, die durch wissenschaftliche Studien mit hoher Evidenz in Hinblick auf ihre Wirksamkeit evaluiert wurden, sind die kognitive Therapie nach Aaron Beck (15) sowie die interpersonelle Therapie nach Gerald Klerman (10). Auch wenn sich dies i.W. auf nur eine Studie stützt, so gilt derzeit als akzeptiert, dass die Pharmakotherapie bei Depression in der Akuttherapie überlegen ist (6). Die antidepressive Wirkung alleiniger Psychotherapie setzt später als diejenige der Pharmakotherapie ein. Einiges spricht für additive Effekte gleichzeitiger Pharmakotherapie kombiniert mit IPT oder CBT in der Akutbehandlung schwererer Depression $(14,16)$.

Bei antidepressiver Therapie ist die Mindestdauer von sechs bis zwölf Monaten bei einer erstmaligen depressiven Episode zu berücksichtigen (Abb. 1). Eine kürzere Therapie ist aufgrund der hohen Rückfallgefahr nicht vertretbar. Bei Patienten mit einer rezidivierenden depressiven Störung oder einem besonders schweren Verlauf (etwa bei Suizidalität oder psychotischen Symptomen) muss eine antidepressive Langzeittherapie durchgeführt werden (19).

Bei unipolarem Verlauf werden in der Regel selektive Serotonin- 


\section{Tab. 1 Spektrum der bipolaren Erkrankungen}

- Bipolar I: Mindestens eine manische Episode

- Bipolar II: Rezidivierende Depressionen mit Hypomanien

- Zyklothymie: Chronische Phasen subsyndromaler Depressionen und Hypomanien

- Rapid Cycling: bis zu vier Phasen im Jahr (13-20\% der Betroffenen), bei Ultra Rapid Cycling wechseln die Phasen innerhalb von Wochen oder Tagen, beim Ultra-ultra Rapid Cycling sogar innerhalb von Stunden

- Gemischte affektive Episoden treten bei 30 bis 40\% der bipolaren Patienten auf, hier sind sowohl Symptome der Depression als auch der Manie gegeben (Mischzustand)

- Bei allen affektiven Phasen im Rahmen einer bipolaren Störung können kurzfristig psychotische Symptome auftreten

Wiederaufnahmehemmer (SSRI) wie Citalopram, Escitalopram, Fluoxetin, Fluvoxamin, Paroxetin und Sertralin aufgrund ihrer Wirksamkeit und guten Verträglichkeit bei

gleichzeitig niedrigen Behandlungskosten als Mittel der ersten Wahl eingesetzt. Bei Unverträglichkeit oder Therapieresistenz selbst in höherer Dosierung und ausrei-

\section{Tab. 2 Diagnostik depressiver Störungen nach ICD-10}

Depressive Episode

(Erste Episode: F32; im Rahmen eines bipolaren Verlaufs: F31; im Rahmen eines unipolaren Ver-

laufs: F33)

- Leicht: 2 Hauptsymptome + 2 Zusatzsymptome

- Mittelgradig: 2 Hauptsymptome + 3-4 Zusatzsymptome

- Schwer: 3 Hauptsymptome + $\geq 4$ Zusatzsymptome

Hauptsymptome

- Depressive Stimmung (ungleich Trauer)

- Interessenverlust, Freudlosigkeit

- Antriebsmangel, erhöhte Ermüdbarkeit

Zusatzsymptome

- Verminderte Konzentration und Aufmerksamkeit

- Vermindertes Selbstwertgefühl und Selbstvertrauen

- Gefühl von Schuld/Wertlosigkeit

- Negative und pessimistische Zukunftsperspektiven

- Suizidgedanken oder -handlungen

- Schlafstörungen

- Verminderter Appetit

Zeitkriterium

- Jeweils Verlauf über $\geq 2$ Wochen

Besondere Verlaufsformen

- Mit somatischen Symptomen: Vorhandensein von mindestens 4 „somatischen“ Symptomen (Interessenverlust, Verlust der Freude an sonst angenehmen Tätigkeiten, mangelnde emotionale Reagibilität auf sonst freudige Ereignisse, frühmorgendliches Erwachen, morgendliches Stimmungstief, psychosomatische Hemmung oder Agitiertheit, deutlicher Appetitverlust, Gewichtsverlust, deutlicher Libidoverlust)

- Schwer, mit psychotischen Symptomen: zusätzliches Vorhandensein von Wahnideen (z.B. Versündigung, Verarmung) und/oder Halluzinationen, (z.B. anklagende Stimmen; Verwesungsgeruch, depressiver Stupor)

\section{Anhaltende affektive Erkrankungen (F34)}

\section{Zyklothymie (F34.0)}

(Anhaltende Stimmungsinstabilität mit zahlreichen Episoden leichter Depression und leicht gehobener Stimmung, die nicht die Schwerekriterien für manische (F30) oder depressive (F32)

Episoden erfüllen)

Dysthymia (F34.1)

(Chronische, gewöhnlich > 2 Jahre anhaltende, milde depressive Verstimmung, die nie oder nur selten („,double Depression“) die Schwerekriterien der depressiven Episoden erfüllt)

chend langer Therapiedauer (die therapeutische Latenz aller Antidepressiva beträgt rund zwei bis drei Wochen) ist ein Wechsel auf eine andere Substanzklasse angezeigt: aus dem psychopharmakologischen Armamentarium seien hier beispielhaft die dualen (serotonergen und noradrenergen) Antidepressiva (Venlafaxin, Duloxetin), der selektive Noradrenalin-Wiederaufnahmehemmer Reboxetin, sedierendanxiolytische Substanzen wie Mirtazapin und Trazodon sowie reversible MAO-Hemmer (Moclobemid) genannt. Trizyklische Antidepressiva sollten aufgrund ihrer höheren Toxizität und schlechteren Verträglichkeit nur bei besonderer Indikation eingesetzt werden (8).

\section{Switch bei bipolaren Patienten vermeiden}

Besondere Berücksichtigung verdienen Patienten mit einer Depression, die im Längsschnitt an einer bipolaren affektiven Störung (Tab. 1) leiden: tatsächlich stellt die Verkennung einer bipolaren Depression als eine unipolare eine der häufigsten Fehldiagnosen dar (7). Das ist deshalb besonders fatal, da eine antidepressive Monotherapie bei dieser Störung aufgrund des hohen Switch-Risikos in eine manische Episode abzulehnen ist. Diese Patienten benötigen eine phasenprophylaktische Therapie als Eckpfeiler ihrer Behandlung, z.B. mit Lamotrigin, Lithium oder Valproinsäure. Jüngst wurde auch die Kombination von Antidepressivum und atypischem Antipsychotikum (z.B. Fluoxetin plus Olanzapin) als potente antidepressive Therapie ohne Risikoerhöhung für einen manischen Switch postuliert (18).

\section{Prognose}

Insgesamt weisen die meisten Formen depressiver Erkrankungen eine gute Prognose auf. Die Remissionsraten liegen in plazebokontrollierten Studien unter 50\% (11, 17). Über die Remissionsraten unter Alltagsbedingungen (Effectiveness) gibt es nur wenige Daten. Montgomery et al. fanden für Venlafaxin vs. Escitalopram nach acht Wochen knapp 80\% Remissionen (12). Auch sogenannten 
„therapierefraktären“ Patienten kann durch Kombination von Therapiestrategien, Anwendung von adjuvanten Verfahren und spezifischen Augmentationsverfahren geholfen werden.

Eine Alternative bei Nichtansprechen einer depressiven Symptomatik auf mehrere suffiziente Therapieversuche ist die Elektrokrampftherapie. Diese Methode, deren Anwendung spezialisierten Zentren vorbehalten ist, zeichnet sich zwar durch ausgezeichnete Wirksamkeit und vergleichsweise geringe Nebenwirkungen aus, doch wird sie teilweise aufgrund von unbegründeten Resentiments und Vorurteilen von Seiten der Patienten, aber auch der Ärzte, zu selten angewendet (5).

\section{Current Treatment of Depression} Depressive states are the most common mental diseases. During the course of their lives some 15 to $20 \%$ of the general population suffer from a depressive episode on at least one occasion. With regard to treatment, a combination of psychotherapeutic and pharmacotherapeutic measures proves to be superior to the respective monotherapy. New pharmacological options are available in the form of the selective serotonin and norepinephrine reuptake inhibitor, duloxetine, and the pure enantiomer selective serotonin reuptake inhibitor escitalopram. When treating bipolar depression, account must be taken of the fact that antidepressants may trigger a switch-over to the manic state, which implies that, whenever necessary, phase prophylactic therapy with mode-stabilizing drugs should be employed.

\section{Key words}

depression - treatment - diagnostic psychotherapy

\section{Literatur}

1. Arsenault-Lapierre G, Kim C, Turecki G. Psychiatric diagnoses in 3275 suicides: a meta-analysis. BMC Psychiatry 2004; 4: 37

2. Avery DH, Holtzheimer III PE, Fawaz W, Russo J, Neumaier J, Dunner DL, Haynor DR, Claypoole KH, Wajdik C, Roy-Byrne P. A Controlled Study of Repetitive Transcranial Magnetic Stimulation in Medication-Resistant Major Depression. Biol Psychiatry 2005; doi: 10.1016/j.biopsych.2005.07.003

\section{Abb. 1 Schematisierte Darstellung des Langzeitverlaufs} einer depressiven Erkrankung

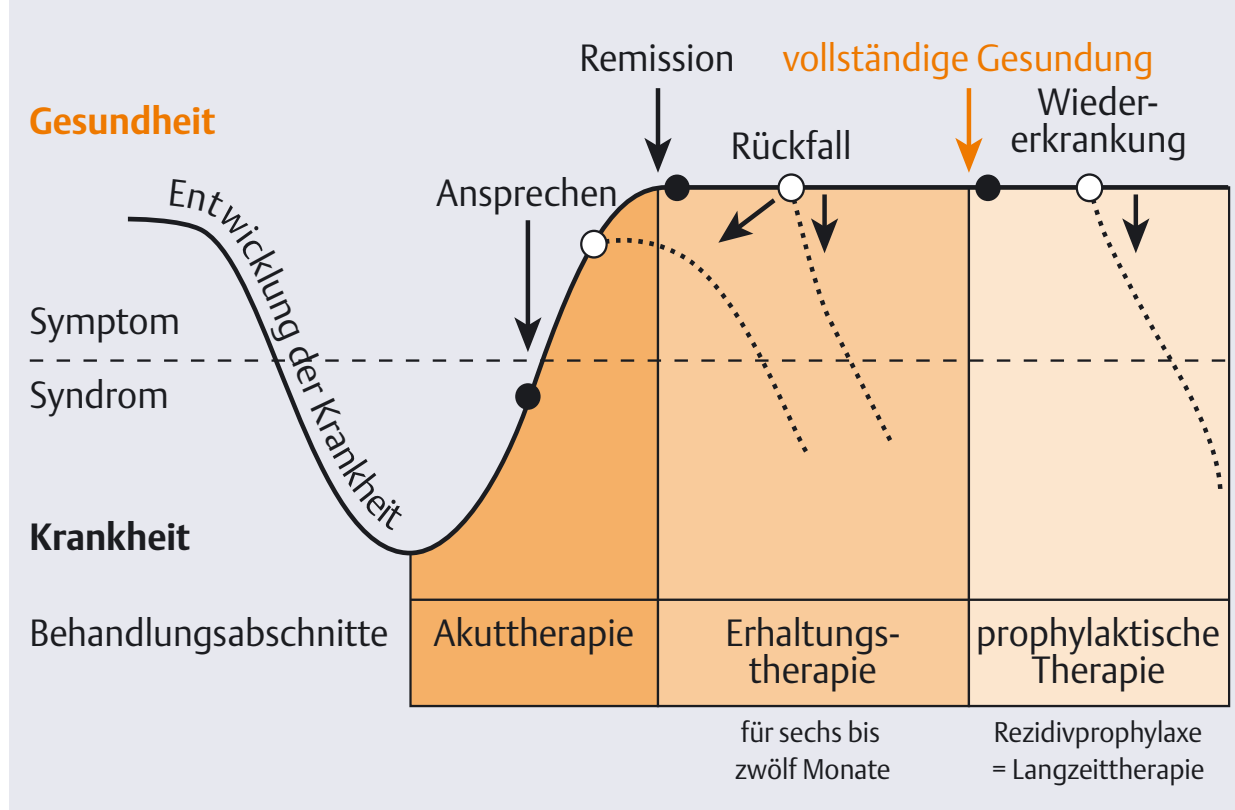

\section{Tab. 3 Symptomatik und Diagnostik (hypo)manischer Episoden nach ICD-10}

F30: Erste Episode ohne vorangegangene manische, hypomanische oder depressive Episode; F31: Im Rahmen einer bipolaren Erkrankung (wenigstens 1 vorangegangene manische, hypomanische oder depressive Episode)

Hypomanie (F30.0, F31.0)

- Anhaltende leicht gehobene Stimmung

- Gesteigerter Antrieb und Aktivität

- Auffallendes Gefühl gesteigerten Wohlbefindens und körperlicher und seelischer Leistungsfähigkeit; häufig zusätzlich: gesteigerte Geselligkeit, Gesprächigkeit, Vertraulichkeit, Libidostörung, vermindertes Schlafbedürfnis

- Alternativ: Reizbarkeit, Selbstüberschätzung, „flegelhaftes“ Verhalten statt Euphorie

- Zeitkriterium: „einige Tage“

- Evtl. auch deutliche Beeinträchtigung der beruflichen oder sozialen Aktivitäten, aber nicht schwer oder vollständig (Manie)

Manie ohne psychotische Symptome (F30.1, F31.1)

- Situationsinadäquat gehobene Stimmung

- Vermehrter Antrieb, Überaktivität

- Rededrang

- Vermindertes Schlafbedürfnis

- Verlust üblicher sozialer Hemmungen

- Starke Ablenkbarkeit, Aufmerksamkeit kann nicht mehr aufrechterhalten werden

- Selbstüberschätzung, freie Äußerung von Größenideen und maßlosem Optimismus

- Riskante Projekte, leichtsinnige Geldausgaben

- Alternativ: Stimmung gereizt und misstrauisch statt euphorisch

- Zeitkriterium: wenigstens 1 Woche

Manie mit psychotischen Symptomen (F30.2, F31.2)

- Selbstüberschätzung/Größenideen wahnhaften Ausmaßes

- Ideenflucht und Rededrang bis zur sprachlichen Unverständlichkeit

- Evtl. Verfolgungswahn (als Resultat von gesteigerter Reizbarkeit/Misstrauen)

- Aggressivität/Gewalttätigkeit

- Evtl. weitere synthyme oder parathyme psychotische Symptome (z.B. Wahngedanken, Halluzinationen) 
3. Blier P. The burden of depression and the challenge of its recognition and treatment. CNS Spectrum 2002; 7: 119

4. DeRubeis RJ, Gelfand LA, Tang TZ, Simons AD. Medications versus cognitive behavior therapy for severely depressed outpatients: mega-analysis of four randomized comparisons. Am J Psychiatr 1999; 156(7): 1007-13

5. Dowman J, Patel A, Rajput K. Electroconvulsive therapy: attitudes and misconceptions. J ECT 2005; 21: 84-87

6. Elkin I, Shea MT, Watkins JT, Imber SD, Sotsky SM, Collins JF, Glass DR, Pilkonis PA, Leber WR, Docherty JP, et al. National Institute of Mental Health Treatment of Depression Collaborative Research Program. General effectiveness of treatments. Arch Gen Psychiatry 1989; 46 (11): 971-82

7. Furukawa TA, Konno W, Morinobu S et al. Course and outcome of depressive episodes: Comparison between bipolar, unipolar and subthreshold depression. Psychiatry Research 2000; 96: 211-220

8. Furukawa TA, McGuire $H$, Barbui $C$. Meta-analysis of effects and side effects of low dosage tricyclic antidepressants in depression: systematic review. BM] 2002; 325 (7371): 991

9. Kessler RC, McGonagle KA, Nelson CB et al. Sex and depression in the National Co- morbidity Survey. II: Cohort effects. Journal of Affective Disorders 1994; 30: 15-26

10. Klerman GL, Weissmann MM. Interpersonal psychotherapy (IPT) and drugs in the treatment of depression. Pharmacopsychiatry $1987 ; 20: 3-7$

11. Lam RW, Kennedy SH. Evidence-based strategies for achieving and sustaining full remission in depression: focus on metaanalyses. Can J Psychiatry 2004; 49 (3 Suppl 1): $175-265$

12. Montgomery SA, Huusom AK, Bothmer J. A randomised study comparing escitalopram with venlafaxine $X R$ in primary care patients with major depressive disorder. Neuropsychobiology 2004; 50 (1): 57-64

13. Murray CJL, Lopez AD (eds.). The global burden of disease and injury series, volume 1: a comprehensive assessment of mortality and disability from diseases, injuries, and risk factors in 1990 and projected to 2020. Cambridge, MA: Published by the Harvard School of Public Health on behalf of the World Health Organization and the World Bank, Harvard University Press, 1996 14. Pampallona S, Bollini P, Tibaldi G, Kupelnick B, Munizza C. Combined pharmacotherapy and psychological treatment for depression: a systematic review. Arch Gen Psychiatry 2004; 61 (7): 714-9

15. Simons AD, Gordon JS, Monroe SM et al.
Toward an integration of psychologic, social, and biologic factors in depression: Effects on outcome and course of cognitive therapy. Journal of Consulting and Clinical Psychology 1995; 63: 369-377

16. Thase ME, Greenhouse JB, Frank E, Reynolds CF 3rd, Pilkonis PA, Hurley K, Grochocinski V, Kupfer DJ.Treatment of major depression with psychotherapy or psychotherapy-pharmacotherapy combinations. Arch Gen Psychiatry 1997; 54(11): 1009-15

17. Thase ME. Achieving remission and managing relapse in depression. J Clin Psychiatry 2003; 64 (Suppl 18): 3-7

18. Tohen M, Vieta E, Calabrese J et al. Efficacy of olanzapine and olanzapine-fluoxetine combination in the treatment of bipolar I depression. Arch Gen Psychiatry 2005; 60 : 1079-1088

19. Winkler D, Tauscher J, Kasper S. Maintenance treatment in depression. Curr Op Psychiatry 2002; 15: 63-68

\section{Korrespondenzadresse:}

Dr. med. univ. Dietmar Winkler

Klinische Abteilung für

Allgemeine Psychiatrie

Medizinische Universität Wien

Währinger Gürtel 18-20

A-1090 Wien, Österreich

dietmar.winkler@meduniwien.ac.at 